\title{
Analysis of corpus callosum size depending on age and sex
}

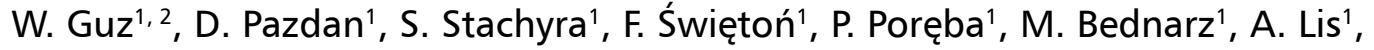 \\ A. Kazańska1, J. Krukowska2, 3, J. Klęba2, 3, A. Urbanik ${ }^{4}$ \\ ${ }^{1}$ Department of Electroradiology, Institute of Nursing and Health Sciences, Faculty of Medicine, University of Rzeszow, \\ Poland \\ ${ }^{2}$ Clinical Department of Radiology and Diagnostic Imaging, the Clinical Voivodship Hospital No. 2, Rzeszow, Poland \\ ${ }^{3}$ Unit of Human Normal Anatomy, Department of Morphological Sciences, Faculty of Medicine, University of Rzeszow, \\ Poland \\ ${ }^{4}$ Department of Radiology, Collegium Medicum, Jagiellonian University, Krakow, Poland
}

[Received: 26 February 2018; Accepted: 18 June 2018]

Background: The aim of the study was to analyse changes in the size of the corpus callosum (CC) depending on age and sex and to establish the reference values of the morphometric indices of the CC in the Polish population.

Materials and methods: The results of magnetic resonance studies of 1108 patients performed in the years 2010-2014 were analysed. Two independent radiologists evaluated cerebral images to exclude deviations from normal state. In patients divided according to sex and to 10 age groups, measurements of CC and brain dimensions were made and morphometric indices were calculated.

Results: The results of measurements related to the following parameters: lengths of longitudinal cross-section of CC (CD), CC thickness in the narrowest place isthmus $(E F)$, the largest linear dimension of the brain from the frontal pole to the occipital pole $(A B)$, the longitudinal cross-section area of the CC (A1) and cerebral cross-section area $(A 2)$ as well as $C D / A B$ and $A 1 / A 2$ ratios are summarised in 7 figures and 3 tables.

Conclusions: It was demonstrated, that in all age groups there are statistically significant differences in the values of the analysed parameters and ratios of CC size. It was indicated, that there are no statistically significant differences between men and women in the CD, EF, and A1 parameters related to CC size, and the profiles of variations of these parameters are very similar. It was proved that there are statistical differences between women and men in parameters/indicators concerning of the brain size. (Folia Morphol 2019; 78, 1: 24-32)

Key words: corpus callosum, magnetic resonance, morphometry, age groups, sex

\section{INTRODUCTION}

The corpus callosum (CC) i.e. commissura magna is a white matter band located at the bottom of the longitudinal fissure of the brain that connects the two hemispheres of the brain. It plays a very important role, allowing information to be transmitted between the right and left hemisphere of the brain, thus integrating their activities and providing the ability to perform complex functions [9]. For this reason, this structure has been frequently and continues to be screened for morphological and morphometric evaluation $[10,19]$ and to determine the correlation of

Address for correspondence: Dr. W. Guz, Department of Electroradiology, Institute of Nursing and Health Sciences, Faculty of Medicine, University of Rzeszow, ul. Prof. A. Krzyżanowskiego 50, 35-329 Rzeszów, Poland, e-mail: wguz@op.pl 
its developmental disorders and pathological states with the occurrence of specific clinical symptoms $[2,17,26]$. The numerous reports link the presence of morphological abnormalities of the corpus callosum with mental disorders, speech dysfunction and dyslexia, seizures [7], autism [12], Alzheimer's disease [15], cognitive processes [22] and other pathologies $[26,27]$. There are also reports linking variants of the shape and size of the CC to gender and age and also to the size of the brain $[1,4-6,8,10,13,14$, $18,19,28,30,32,33]$. The importance of researches on morphology and the function of CC underline the fact that in 2012 a separate organisation dedicated to the development of researches to understand the role of this structure and to help people with developmental disorders of the CC was invoked - National Organisation for Disorders of the Corpus Callosum (NODCC) [24].

The aim of the study was to analyse the changes of CC size depending on age and sex and to establish the reference values of morphometric indicators concerning $\mathrm{CC}$ in the Polish population.

\section{MATERIALS AND METHODS}

The analysis was performed on 1108 patients, including 529 women and 579 men magnetic resonance (MR) studies results, performed in the Magnetic Resonance Unit in the years 2010-2014, diagnosed with scanner Philips Achieva 1.5T. All of these individuals, on the basis of the information contained in the referral, had no central nervous system (CNS) injury or CNS diseases or pathology. In each patient also performed a morphological evaluation of the brain image with particular emphasis on the corpus callosum; the evaluation was performed by two radiologists independently evaluated without finding any deviation from the normal state. Patients were divided into 10 age groups (0-5/6-10/11-15/1620/21-30/31-40/41-50/51-60/61-70/> 70), in each group. The men:women (M:W) ratios were close to $1: 1$, with the exception of $0-5 / 6-10$ and $11-15$, where men predominated (95:57/71:53/65:59/50:57/51:51/ 50:50/50:51/50:62/52:50/50:50).

Corpus callosum measurements were obtained based on MR studies performed in the frFSE sequences in T2-dependent images in sagittal plane, along the midline of the head (midsagittal).

The following linear measurements and surface areas were performed in sagittal plane of the brain (Fig. 1A, B):

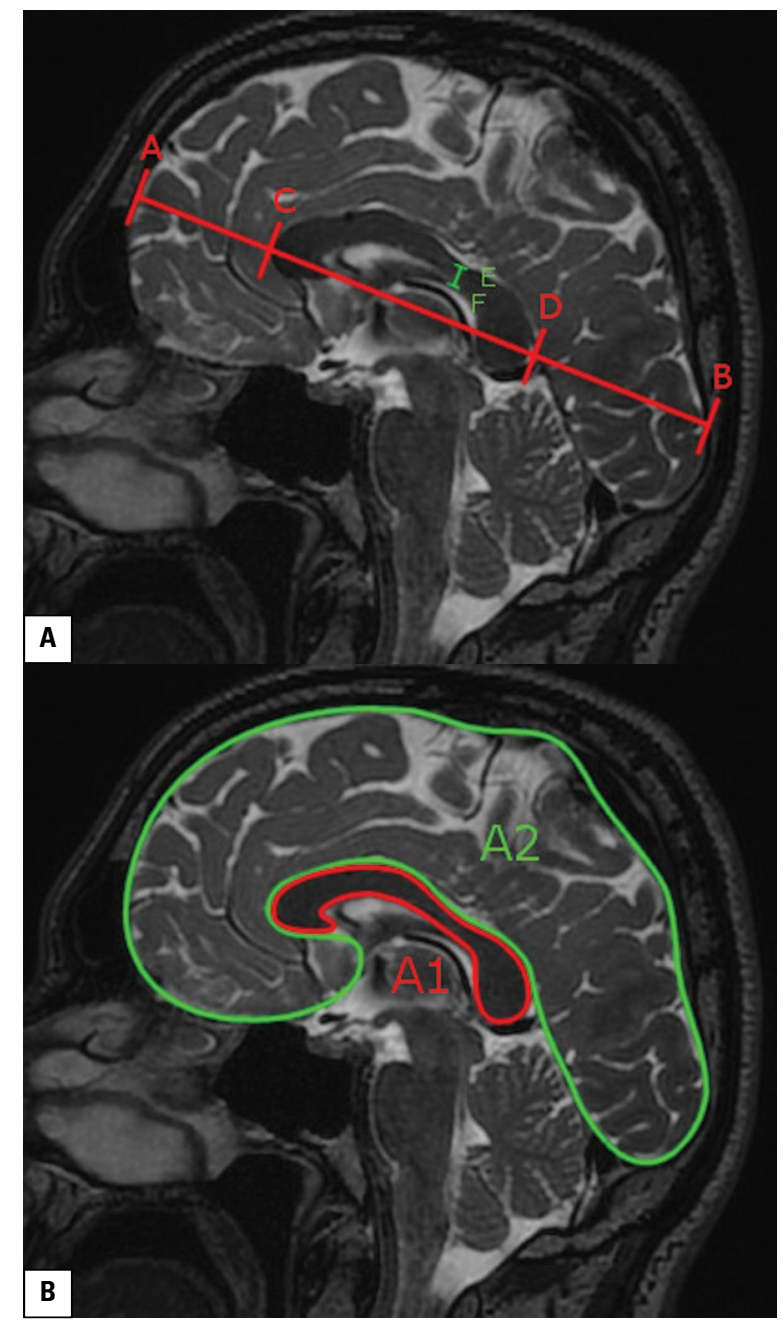

Figure 1. A, B. Scheme of linear measurements and areas of corpus callosum and brain.

- CD - length of longitudinal section of corpus callosum;

- EF - thickness of corpus callosum in the narrowest place - isthmus;

$-A B-$ largest linear dimension of the brain on the level $C D$.

Measurements of the cross-sectional areas:

- A1 - cross-sectional area of corpus callosum in midline plane (midsagittal);

- A2 - cross-sectional area of the brain in midline plane (midsagittal).

In addition, the parameters expressing relative ratios of measured structures were added. The introduction of these parameters was aimed obtaining indicators independent from the dimensions of the head/brain. 


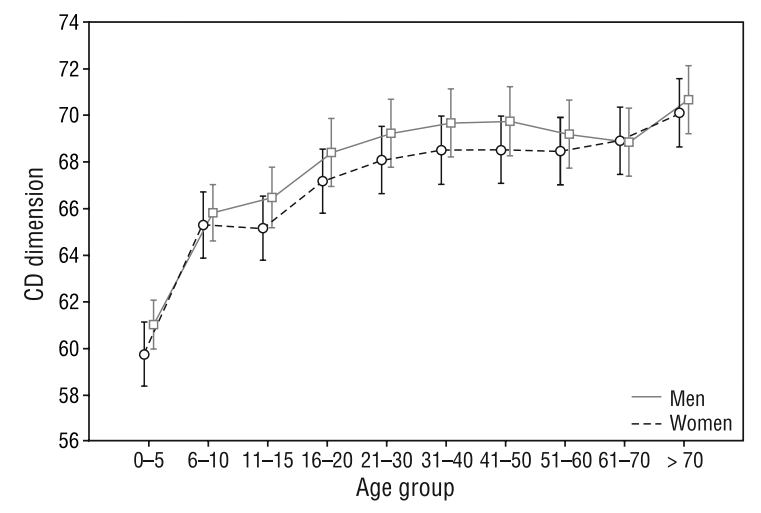

Figure 2. CD value in age groups, depending on sex.

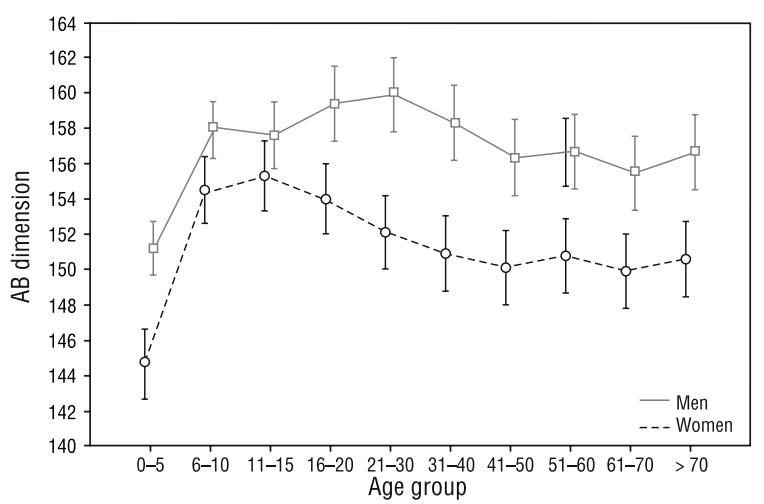

Figure 3. Values of $A B$ parameter in age groups, depending on sex.

The following relative ratios were calculated:

- A1/A2 - i.e. the ratio of the area of the cross-sectional area of corpus callosum to the area of the cerebral cross-sectional area in the midline;

- $C D / A B$ - i.e. the ratio of the length of the corpus callosum to the length of the brain in the sagittal section of the midline.

The collected material was subjected to statistical analysis. The analysis was based on a two-way analysis of variance, Tukey's HSD test, and a non-parametric analysis at the final stage using the Kruskal-Wallis ANOVA test.

\section{RESULTS}

Figures 2-8 and Tables $1-3$ show selected, important parameters of this analysis. The CD parameter (length of the longitudinal section of CC) was analysed by sex and subsequent age groups. Results are shown in Figure 2.

The analysis of the $C D$ parameter shows that this value varies with age, wherein for the size of $\mathrm{CC}$ rapid increase in the age of 0-10 is characteristic, followed by a short plateau phase over a period of 11-15 years, then

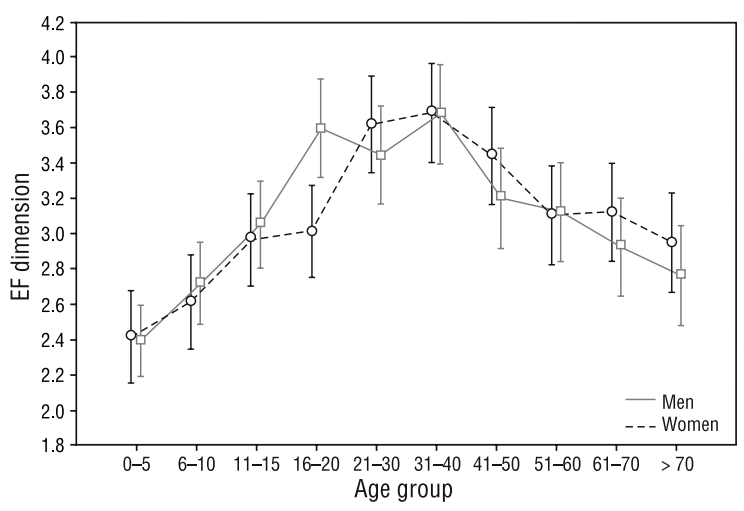

Figure 4. Values of EF parameter in age groups, depending on sex.

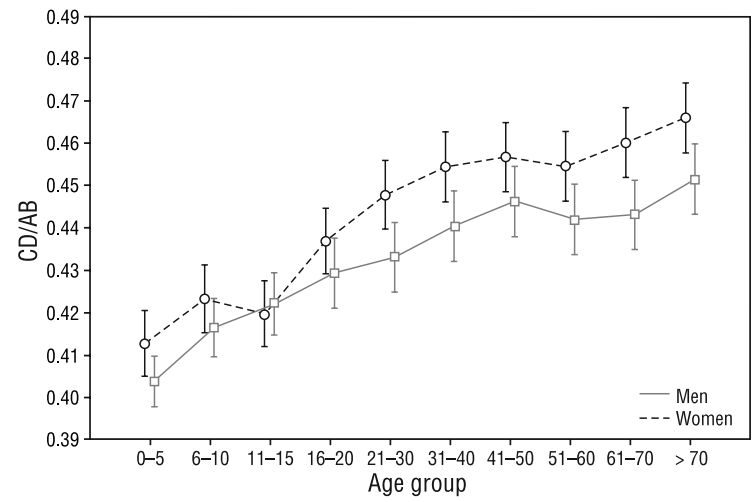

Figure 5. Value of $C D / A B$ parameter in age groups, depending on sex.

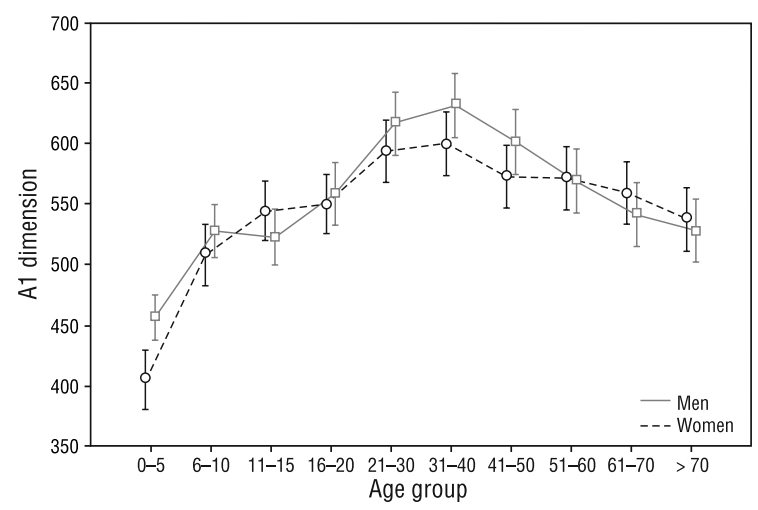

Figure 6. Value of $\mathrm{A} 1$ parameter in age groups, depending on sex.

the parameter values gradually increase to achieve the peak in a period of $41-50$ years of age. After 50 years of age, this parameter gradually decreases to increase again in patients over 70 years of age. There are also differences in $C D$ parameter values depending on sex. There is no difference in CD values in the ages of $0-10$ whereas between 11 and 70 years of age the value of this parameter is slightly higher for men than for women, but these differences are not statistically significant. 


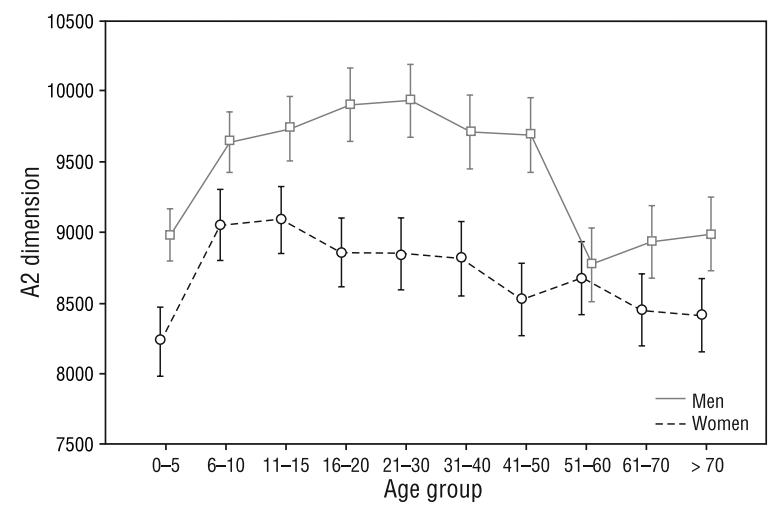

Figure 7. Value of $A 2$ parameter in age groups, depending on sex.

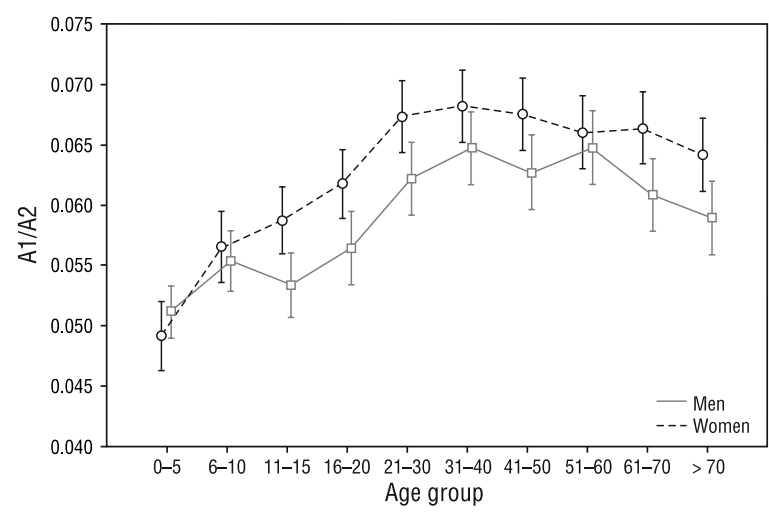

Figure 8. Value of $A 1 / A 2$ parameter in age groups, depending on sex.

The $A B$ parameter (the largest linear dimension of the brain from frontal to occipital) was analysed by sex and subsequent age groups. The results are summarised in Figure 3.

The value of $A B$ parameters rapidly increases in the period of $0-10$ years of age in both men and women.
Then we observe a quiet growth phase up to 15 years of age in women and up to 30 years of age in men followed by a decrease in the value of $A B$ parameter in both sexes of similar severity during the $50 \mathrm{~s}$. It is worth noting that the value of the $A B$ parameter for 41-50 years of age in both sexes is half the value of this parameter from 0-10 years of age. In the period of 51-70 years of age $A B$ parameter values in both sexes remain stable. Over the lifetime, the values of $A B$ parameter are higher for men than for women, which is statistically significant.

The analysis of the EF parameter (thickness of CC in the narrowest place - isthmus) was performed on the basis of sex and age groups. The results are summarised in Figure 4.

For the EF parameter, there is a gradual increase in its value in both sexes up to 40 years of age, followed by a gradual decline in its value over the age of 70, while the EF value for both sexes in the age group above 70 years of age corresponds to its value in the age of 10-15 years. It is worth adding that in the period 15-20 years the absolute value of the EF parameter is higher in men, while in the period 21-30 years and $51-70$ years it is higher in women, which is not statistically significant. Between $0-15$ years of age and 31-40 years of age the values of this parameter in both sexes are very similar.

$A C D / A B$ index corresponding to the ratio of the $C C$ length to the length of the brain in cross-sectional area in the midline was generated and analysed according to sex and age groups. The results are summarised in Figure 5

The $C D / A B$ indicator shows gradual linear growth in subsequent age groups in both sexes. Only in the period of 11-15 years of age and 41-60 years of age

Table 1. Statistical differences of parameters values depending on sex — nonparametric approach

\begin{tabular}{|c|c|c|c|c|c|c|c|c|c|}
\hline \multirow[t]{2}{*}{ Variable } & \multicolumn{2}{|c|}{ Sum of ranks } & \multirow[t]{2}{*}{$\mathbf{U}$} & \multirow[t]{2}{*}{ Z } & \multirow[t]{2}{*}{$\mathbf{p}$} & \multirow{2}{*}{$\begin{array}{c}\text { Z without } \\
\text { margin values }\end{array}$} & \multirow[t]{2}{*}{ 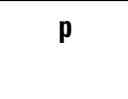 } & \multicolumn{2}{|c|}{ N weight } \\
\hline & Women & Man & & & & & & Women & Men \\
\hline$C D$ & 280513.5 & 318451.5 & 144532.5 & -0.9069 & 0.364449 & -0.9069 & 0.364436 & 521 & 573 \\
\hline EF & 288966.0 & 308905.0 & 144454.0 & 0.8683 & 0.385231 & 0.8688 & 0.384984 & 520 & 573 \\
\hline$A B$ & 231242.0 & 367723.0 & 95261.0 & -10.3472 & 0.000000 & -10.3473 & 0.000000 & 521 & 573 \\
\hline $\mathrm{A} 1$ & 283952.5 & 315012.5 & 147971.5 & -0.2480 & 0.804118 & -0.2480 & 0.804118 & 521 & 573 \\
\hline A2 & 220514.5 & 378450.5 & 84533.5 & -12.4025 & 0.000000 & -12.4025 & 0.000000 & 521 & 573 \\
\hline $\mathrm{A} 1 / \mathrm{A} 2$ & 255737.5 & 343227.5 & 119756.5 & -5.6539 & 0.000000 & -5.6539 & 0.000000 & 521 & 573 \\
\hline$C D / A B$ & 319203.0 & 279762.0 & 115311.0 & 6.5057 & 0.000000 & 6.5057 & 0.000000 & 521 & 573 \\
\hline
\end{tabular}

Abbreviations - see text. U Mann-Whitney Test (with the correction to continuity) (Total base) in relation to variable: sex. Marked results are significant with $\mathrm{p}<0.05000$. Disregard the incidents: 35; 277; 297; 311; 357; 367; 445; 482; 634; 684; 766; 856; 886; 1031 
Table 2. Mean values (mv) and standard deviations (sd) of the analysed parameters and indexes divided for age groups in women. The values are presented in $\mathrm{mm}$ for $\mathrm{CD}, \mathrm{EF}, \mathrm{AB}$ measurements, in $\mathrm{mm}^{2}$ for $\mathrm{A} 1$ and $\mathrm{A} 2$ measurements

\begin{tabular}{|c|c|c|c|c|c|c|c|c|c|c|}
\hline \multirow[t]{2}{*}{ Parameters } & \multicolumn{10}{|c|}{ Age groups } \\
\hline & $<5$ & $5-10$ & $11-15$ & $16-20$ & $21-30$ & $31-40$ & $41-50$ & $51-60$ & $61-70$ & $>70$ \\
\hline $\mathrm{CD}-\mathrm{mv}$ & 59.89 & 65.31 & 65.29 & 67.17 & 68.08 & 68.49 & 68.54 & 68.47 & 68.93 & 70.05 \\
\hline$C D$-sd & 6.0 & 5.1 & 7.0 & 4.0 & 4.0 & 6.0 & 5.4 & 4.6 & 4.5 & 4.7 \\
\hline$E F-m v$ & 2.41 & 2.62 & 2.95 & 3.01 & 3.62 & 3.65 & 3.44 & 3.17 & 3.12 & 2.97 \\
\hline$E F-s d$ & 0.9 & 1.1 & 1.0 & 0.9 & 0.8 & 1.0 & 1.1 & 1.1 & 1.0 & 0.8 \\
\hline$A B-m v$ & 144.80 & 154.34 & 155.46 & 153.90 & 152.12 & 150.97 & 150.13 & 150.96 & 149.90 & 150.54 \\
\hline$A B-s d$ & 9.4 & 8.2 & 8.5 & 7.2 & 7.4 & 6.1 & 6.9 & 7.7 & 6.1 & 6.7 \\
\hline $\mathrm{A} 1-\mathrm{mv}$ & 404.38 & 507.63 & 540.89 & 549.58 & 593.38 & 598.30 & 572.17 & 572.06 & 558.60 & 537.62 \\
\hline $\mathrm{A} 1-\mathrm{sd}$ & 102.86 & 78.04 & 97.80 & 79.48 & 101.97 & 91.80 & 100.05 & 130.80 & 107.17 & 89.44 \\
\hline $\mathrm{A} 2-\mathrm{mv}$ & 8240.20 & 9048.00 & 9119.40 & 8863.30 & 8846.40 & 8804.50 & 8522.20 & 8675.10 & 8449.40 & 8403.70 \\
\hline$A 2-s d$ & 1050.1 & 910.2 & 1422.2 & 1464.6 & 763.8 & 674.0 & 790.7 & 311.5 & 737.2 & 766.2 \\
\hline $\mathrm{A} 1 / \mathrm{A} 2-\mathrm{mv}$ & 0.047 & 0.055 & 0.057 & 0.061 & 0.065 & 0.066 & 0.065 & 0.066 & 0.063 & 0.063 \\
\hline $\mathrm{A} 1 / \mathrm{A} 2-\mathrm{sd}$ & 0.011 & 0.009 & 0.015 & 0.014 & 0.013 & 0.011 & 0.013 & 0.005 & 0.017 & 0.011 \\
\hline $\mathrm{CD} / \mathrm{AB}-\mathrm{mv}$ & 0.41 & 0.42 & 0.42 & 0.44 & 0.45 & 0.45 & 0.46 & 0.45 & 0.46 & 0.47 \\
\hline$C D / A B-s d$ & 0.029 & 0.027 & 0.041 & 0.027 & 0.022 & 0.040 & 0.031 & 0.027 & 0.028 & 0.030 \\
\hline
\end{tabular}

Abbreviations - see text

Table 3. Mean values (mv) and standard deviations (sd) of the analysed parameters and indexes divided for age groups in men. The values are presented in $\mathrm{mm}$ for $\mathrm{CD}, \mathrm{EF}, \mathrm{AB}$ measurements, in $\mathrm{mm}^{2}$ for $\mathrm{A} 1$ and $A 2$ measurements

\begin{tabular}{lcccccccccc}
\hline Parameters & \multicolumn{10}{c}{ Age groups } \\
\hline & $\mathbf{5}$ & $\mathbf{5 - 1 0}$ & $\mathbf{1 1 - 1 5}$ & $\mathbf{1 6 - 2 0}$ & $\mathbf{2 1 - 3 0}$ & $\mathbf{3 1 - 4 0}$ & $\mathbf{4 1 - 5 0}$ & $\mathbf{5 1 - 6 0}$ & $\mathbf{6 1 - 7 0}$ & $>\mathbf{7 0}$ \\
\hline $\mathrm{CD}-\mathrm{mv}$ & 61.03 & 65.83 & 66.45 & 68.59 & 69.24 & 69.63 & 69.85 & 69.43 & 68.84 & 70.68 \\
$\mathrm{CD}-\mathrm{sd}$ & 5.7 & 5.8 & 4.6 & 6.2 & 5.4 & 5.0 & 4.7 & 3.7 & 4.8 & 4.9 \\
EF-mv & 2.40 & 2.72 & 3.04 & 3.60 & 3.45 & 3.66 & 3.23 & 3.13 & 2.93 & 2.76 \\
EF-sd & 1.0 & 1.0 & 1.1 & 0.9 & 1.0 & 0.8 & 1.2 & 1.2 & 1.1 & 0.9 \\
$\mathrm{AB}-\mathrm{mv}$ & 151.17 & 158.03 & 157.35 & 159.39 & 159.87 & 158.17 & 156.27 & 156.77 & 155.43 & 156.62 \\
$\mathrm{AB}-\mathrm{sd}$ & 8.0 & 7.5 & 8.8 & 9.1 & 7.1 & 7.8 & 6.4 & 6.0 & 7.0 & 6.4 \\
$\mathrm{~A} 1-\mathrm{mv}$ & 456.15 & 527.48 & 522.40 & 559.67 & 616.43 & 628.07 & 785.57 & 567.79 & 540.85 & 527.60 \\
$\mathrm{~A} 1-\mathrm{sd}$ & 113.12 & 91.10 & 93.19 & 108.52 & 89.57 & 83.33 & 102.28 & 110.03 & 112.12 & 95.48 \\
$\mathrm{~A} 2-\mathrm{mv}$ & 8980.19 & 9636.24 & 9716.23 & 9900.90 & 9932.21 & 9710.74 & 9681.67 & 8772.54 & 8931.23 & 8983.86 \\
$\mathrm{~A} 2-\mathrm{sd}$ & 1043.5 & 1069.4 & 1336.4 & 730.6 & 543.7 & 707.2 & 703.7 & 242.7 & 776.6 & 705.6 \\
$\mathrm{~A} 1 / \mathrm{A} 2-\mathrm{mv}$ & 0.048 & 0.053 & 0.052 & 0.055 & 0.061 & 0.064 & 0.062 & 0.065 & 0.058 & 0.057 \\
$\mathrm{~A} 1 / \mathrm{A} 2-\mathrm{sd}$ & 0.012 & 0.011 & 0.014 & 0.010 & 0.010 & 0.008 & 0.013 & 0.004 & 0.015 & 0.011 \\
$\mathrm{CD} / \mathrm{AB}-\mathrm{mv}$ & 0.40 & 0.42 & 0.42 & 0.43 & 0.43 & 0.44 & 0.45 & 0.44 & 0.44 & 0.45 \\
$\mathrm{CD} / \mathrm{AB}-\mathrm{sd}$ & 0.032 & 0.031 & 0.024 & 0.032 & 0.029 & 0.027 & 0.026 & 0.023 & 0.028 & 0.029 \\
\hline
\end{tabular}

Abbreviations - see text

in women and $41-70$ years of age in men its value stabilises. In all age groups except 11-15 years, the value of this parameter in women is higher than in men, which is statistically significant.
The A1 parameter (cross sectional area of CC in cross sectional area in the plane passing through the midline) was analysed by sex and subsequent age groups. The results are summarised in Figure 6. 
Similarly to the EF parameter, the A1 parameter reaches its peak in both sexes in the 31-40 years of age group, and the final value in the $>70$ year of age group in both sexes corresponds to that of the 11-15 years of age period. The value of this parameter is higher in men in age groups $0-5$ years and 21-50 years, and in women aged $11-15$ and $60-70$ years, which is not statistically significant.

The analysis of the A2 parameter (area of the cerebral cross section in the plane passing through the midline) was performed, depending on sex and in subsequent age groups. The results are summarised in Figure 7.

The changes of $A 2$ parameter values in the following age groups were similar to those of the $A B$ parameter. The phase of rapid growth in the period of 0-10 years of age can be distinguished, followed by the quiet growth phase, which reaches peak in 11-15 years of age in women and in 21-30 years of age in men. In subsequent age groups, the $A 2$ value gradually decreases, with men experiencing a rapid decline in the 51-60 years of age group and a slightly lower decrease in the 41-50 years of age group. After a short stabilisation of $\mathrm{A} 2$ parameter in women within the age of $41-50$ years, its value gradually decreases until the age over 70 years. In men, the value of this parameter gradually slightly increases between 51 and 70 years of age. Over the lifetime, the absolute value of $\mathrm{A} 2$ parameter is higher in men than in women, which is statistically significant, and the differences are the highest in the age range of 16-50 years. In the age range of 51-60 years, $A B$ parameter values approach to each other in both sexes, and in the subsequent age groups the $A B$ parameter value in men gradually increases.

The $A 1 / A 2$ index (the ratio of cross-sectional area of CC to cross-sectional area of the brain in the midline) was calculated and analysed depending on sex and subsequent age groups. The results are summarised in Figure 8.

The $A 1 / A 2$ index values show a gradual increase in the age group of 0-40 years, with linear growth in women and with temporary stabilisation in men over 11-20 years of age. In both sexes, the stabilisation of the value of this parameter is visible in the period of 41-60 years of age, whereas in the subsequent age groups a slight decrease occurs. The absolute value of the A1/A2 index in all age groups (except $0-5$ ) is higher in women than in men, which is statistically significant.
In all age groups, there are statistically significant differences in all analysed measurements in the parametric and nonparametric approach. On the other hand, within the analysed differences concerning sex, insignificant differences for sex in $C D, E F$ and $A 1$ measurements were demonstrated in the nonparametric approach. For all other parameters ( $A B, A 2, A 1 /$ $/ A 2, C D / A B)$ the sex differences in the nonparametric approach were statistically significant, as illustrated in the Table 1.

The range of the reference values (mean values and standard deviation) for the performed measurements and calculated indexes in the age groups and for women and men are presented in the Tables 2 and 3 .

\section{DISCUSSION}

The aim of the study was to analyse changes in the size of the $\mathrm{CC}$, depending on age and sex. The point was to determine the morphometric parameters that are useful in the radiologist's day-to-day operation when assessing the CC. Many publications refer to the varied pathology of the CC and analyse the correlations of its disorder with certain diseases, but the works on population norms related to the size of the $\mathrm{CC}$ is less common in the literature. Some previous works showed greater $[6,13,14,28]$ or similar $[1,4,5,16,33]$ size of CC in women compared to men. In these works $[5,6,14,16,28,30,33]$, the area of $\mathrm{CC}$ in the midline plane (midsagittal), was measured, which was a prerequisite for performing similar measurements in our work. Measurement of CC volume is much more difficult due to the difficulty of precisely defining the CC side limits and requires the use of dedicated software, which, although available, it is not widely used [18]. In own elaboration, the focus was on simple linear measurements and CC surface measurements, also demonstrating some correlations with the sex differentials of these parameters. It was demonstrated, that there are no statistically significant differences between women and men in terms of parameters related to the size of the $\mathrm{CC}$, such as the length of its sagittal cross section (CD), the thickness in the isthmus (EF), or the area of the sagittal cross section (A1), which is consistent with previous works on CC morphometry $[6,11,20,21$, 32]. Lee et al. [18] measured the volume of $\mathrm{CC}$ in the 20-year-old and 40-year-old groups of women and men in the Korean population, showing a significantly greater volume of $\mathrm{CC}$ in men than in women in the 
analysed age groups while there was no significant difference in $\mathrm{CC}$ volume between the 20-year-old and 40-year-old groups [16]. Takeda et al. [32] conclude that, like other authors, they did not show sex differences in measured CC size indexes in the Japanese population. The meta-analysis by Dreisem and Raz [6], describing 43 works published in the twentieth century, has shown that the area of CC is indeed higher in men than in women; however, the area of CC cross-section in relation to cerebral size was higher in women than in men. A similar conclusion can be drawn from Mitchell's study and the results obtained in our study, where the ratio of the area of the CC cross-section to the area of the cerebral cross-section (A1/A2), in midsagittal plane is higher in women, similarly as the length of $C C$ cross-section in relation to the length of the brain cross-section (CD/AB) in this plane [20]. The interesting work by Tanaka-Arakawa et al. [31] indicates the dynamic and non-linear increase in dimensions of individual CC areas during the first 2 years, indicating the existing sex differences in the rate of development of individual $\mathrm{CC}$ parts during this period of life. Our study also found that in both sexes dynamic development of CC over a period of 0-10 years of age; however, the analysed parameters of CC size did not show significant differences between sexes, which is probably related to averaging measurements in the $0-5$ age group. Suganthy et al. [29] in 2003 studied the Indian population for differentiation of CC parameters. One aspect of the study was the assessment of the CC (CD) cross sectional length in the age groups, which was found to be statistically significantly higher in men (tested groups: 18-40, 40-60 and > 60 years of age) than in women. In our work, this tendency was evident; however, in the statistical analysis it was not significant in particular age groups. An interesting issue that had not been unambiguously explained was the fact that the $C D$ parameter increased in the age group over 70 years of age. While initially fast (up to 10 years) and then mild increase in this parameter (up to 50 years of age) can be explained by the increasing amount of nerve fibres within CC and interhemispheric connections, which after 50 years of age begin to decline, it is the fact that in the age group over 70 years of age again gaining higher value is probably due to the atrophic processes of the other brain structures, as a result of which the shape and morphology of the CC change. Similarly as in our study, gradual slight elongation in CC with age was observed and no significant sex differences in CC (A1) surface area. Also Mohammadi et al. [21] in the work on the Iranian population showed differences in the cross-sectional length of the CC (CD), but they were not statistically significant. Due to the differences in the assessed age groups in individual studies $[11,18,20,21,29,31]$, the exact comparative assessment of the $C D$ parameter in different populations is not reliable. In the work of 2015, Prendergast et al. [25] performed a detailed analysis of the changes in $\mathrm{CC}$ region size (described by Witelson [34]) based on age and sex, finding differences between men and women only in the size of the genu of CC. Also in Mourgel et al's [23] work concerning the Greek population, although a small 41-member group, no statistically significant differences in $C D$ dimension of CC between age groups of 45-65 and 66-80 years of age were found. In this work, the dimensions of $\mathrm{CC}$ were compared with the brain sizes in midsagittal plane, which led to a conclusion that these dimensions do not significantly change during life (age groups 24-80). Also the CD/AB ratio was analysed in our material, which value in patients group up to 50 years of age increased linearly and stabilised in the age group of 51-70. The variability of the CD/ $A B$ indicator is most likely due to the rapid growth of CC size in comparison with the other structures of the brain in the period of $0-50$ years of age, and subsequent stabilisation of its value may be associated with cerebral atrophy. Arkedani et al. [3] in his work of 2013 proves that after a statistical correction of the size of the brain, the A1 (CCA) dimension in women is greater than in men. This conclusion was not found statistically significant in our analysis. Analysis of parameters related to brain size $(A B, A 2, C D / A B$ and $A 1 / A 2)$ showed that there is statistically significant difference between women and men, where the absolute values of $A B$ and $A 2$ parameters are higher in men and $C D / A B$ and $A 1 / A 2$ indexes are higher in women, so the sizes of the $\mathrm{CC}$ in women in relation to dimensions are relatively greater. Similar results related to brain size can be found in the work of other authors $[13,20,23]$. Moreover our analysis found that the values of parameters related to brain size $(A B, A 2)$ rapidly increased in age groups of $0-10$ years, which reflects a rapid increase in brain size in this life time. There are also interesting differences in the peak in both parameters $(A B, A 2)$ between men (21-30 years) and women (11-15 years), suggesting that the brain in women reach their target size earlier than in men. 


\section{CONCLUSIONS}

1. In all age groups, there are statistically significant differences in the values of the analysed parameters and their ratios to the size of the CC and brain - what means, that the size of the CC varies considerably throughout human life.

2. There are statistically significant differences between women and men in terms of the following parameters and indicators $A B, A 2, A 1 / A 2, C D / A B$.

3. There are no statistically significant differences between women and men in the measurements of corpus callosum: $C D, E F$ and $A 1$, and the variation profiles of these parameters depending on age in women and men are very similar.

4. The length of sagittal cross-section of corpus callosum (CD) in the period of 0-10 years of age increases rapidly and then grows slowly reaching a peak of 41-50 years of age and grows again slowly in the age group over 70 years.

5. The thickness of corpus callosum in the narrowest place (EF) reaches the peak within the period 30-40 years of age, and then gradually decreases, reaching in the age group over 70 years the size as within the period of 11-15 years of age.

6. The cross-section area of corpus callosum (A1) rapidly increases to 10 year of age, reaches the peak of its size within the period of 31-40 years of age and then gradually decreases, to finally reach in the age over 70 the size at the level of 11-15 years of age.

7. $A B$ and $A 2$ parameters related to brain size reach the peak of its size in different period in women (11-15 years of age) and in men (21-30 years of age).

\section{REFERENCES}

1. Allen LS, Richey MF, Chai YM, et al. Sex differences in the corpus callosum of the living human being. J Neurosci. 1991; 11(4): 933-942, indexed in Pubmed: 2010816.

2. Andronikou S, Pillay T, Gabuza L, et al. Corpus callosum thickness in children: an MR pattern-recognition approach on the midsagittal image. Pediatr Radiol. 2015; 45(2): 258-272, doi: 10.1007/s00247-014-2998-9, indexed in Pubmed: 25173405.

3. Ardekani BA, Figarsky K, Sidtis JJ. Sexual dimorphism in the human corpus callosum: an MRI study using the OASIS brain database. Cereb Cortex. 2013; 23(10): 2514-2520, doi: 10.1093/cercor/bhs253, indexed in Pubmed: 22891036.

4. Byne W, Bleier R, Houston L. Variations in human corpus callosum do not predict gender: a study using magnetic resonance imaging. Behav Neurosci. 1988; 102(2): 222-227, indexed in Pubmed: 3365317.
5. Constant D, Ruther H. Sexual dimorphism in the human corpus callosum? A comparison of methodologies. Brain Res. 1996; 727(1-2): 99-106, indexed in Pubmed: 8842387.

6. Driesem NR, Raz N. The influence of sex, age, and handedness on corpus callosum morphology : a meta-analysis. Psychobiology. 1995; 23(3): 240-247.

7. Firat $A$, Tascioglu $A B$, Demiryurek MD, et al. Evaluation of corpus callosum morphometry in patients with mesial temporal lobe epilepsy with hippocampal sclerosis. Surg Radiol Anat. 2014; 36(1): 47-54, doi: 10.1007/s00276013-1144-y, indexed in Pubmed: 23771402.

8. Franklin MS, Kraemer GW, Shelton SE, et al. Gender differences in brain volume and size of corpus callosum and amygdala of rhesus monkey measured from MRI images. Brain Res. 2000; 852(2): 263-267, indexed in Pubmed: 10678751.

9. Gazzaniga MS. Cerebral specialization and interhemispheric communication: does the corpus callosum enable the human condition? Brain. 2000; 123 ( Pt 7): 1293-1326, indexed in Pubmed: 10869045.

10. Giedd JN, Blumenthal J, Jeffries NO, et al. Development of the human corpus callosum during childhood and adolescence: a longitudinal MRI study. Prog Neuropsychopharmacol Biol Psychiatry. 1999; 23(4): 571-588, indexed in Pubmed: 10390717.

11. Gupta T, Singh B, Kapoor K, et al. Normative data of corpus callosal morphology in a North-West Indian population- an autopsy and MRI study. JNMA J Nepal Med Assoc. 2009; 48(173): 46-51, indexed in Pubmed: 19529058.

12. Hanaie R, Mohri I, Kagitani-Shimono K, et al. Abnormal corpus callosum connectivity, socio-communicative deficits, and motor deficits in children with autism spectrum disorder: a diffusion tensor imaging study. J Autism Dev Disord. 2014; 44(9): 2209-2220, doi: 10.1007/s10803014-2096-8, indexed in Pubmed: 24710811.

13. Jäncke L, Staiger JF, Schlaug G, et al. The relationship between corpus callosum size and forebrain volume. Cereb Cortex. 1997; 7(1): 48-56, indexed in Pubmed: 9023431.

14. Johnson SC, Farnworth T, Pinkston JB, et al. Corpus callosum surface area across the human adult life span: effect of age and gender. Brain Res Bull. 1994; 35(4): 373-377, indexed in Pubmed: 7850489.

15. Kabay SC, Gulbandilar E, Ozden H, et al. Evaluation of the size and area of the corpus callosum with the Osiris method in Alzheimer's disease. Neurodegener Dis. 2009; 6(4): 148-153, doi: 10.1159/000225375, indexed in Pubmed: 19521062.

16. Kertesz A, Polk M, Black SE, et al. Sex, handedness, and the morphometry of cerebral asymmetries on magnetic resonance imaging. Brain Res. 1990; 530(1): 40-48, indexed in Pubmed: 2271951.

17. Krupa K, Bekiesinska-Figatowska M. Congenital and acquired abnormalities of the corpus callosum: a pictorial essay. Biomed Res Int. 2013; 2013: 265619, doi: 10.1155/2013/265619, indexed in Pubmed: 24027754.

18. Lee BY, Sohn JH, Choi MH, et al. A volumetric study of the corpus callosum in 20s and 40s Korean people. Brain Struct Funct. 2009; 213(4-5): 463-467, doi: 10.1007/s00429009-0209-5, indexed in Pubmed: 19597840. 
19. Luders E, Thompson PM, Toga AW. The development of the corpus callosum in the healthy human brain. J Neuroscience. 2010; 30(33): 10985-10990, doi: 10.1523/ jneurosci.5122-09.2010.

20. Mitchell TN, Free SL, Merschhemke M, et al. Reliable callosal measurement: population normative data confirm sex-related differences. AJNR Am J Neuroradiol. 2003; 24(3): 410-418, indexed in Pubmed: 12637291.

21. Mohammadi MR, Zhand P, Mortazavi Moghadam B, et al. Measurement of the corpus callosum using magnetic resonance imaging in the north of iran. Iran J Radiol. 2011; 8(4): 218-223, doi: 10.5812/iranjradiol.4495, indexed in Pubmed: 23329944.

22. Moreno MB, Concha L, González-Santos L, et al. Correlation between corpus callosum sub-segmental area and cognitive processes in school-age children. PLoS One. 2014; 9(8): e104549, doi: 10.1371/journal.pone.0104549, indexed in Pubmed: 25170897.

23. Mourgela S, Anagnostopoulou S, Sakellaropoulos A, et al. An MRI study of sex-and age-related differences in the dimensions of the corpus callosum and brain. Neuroanatomy. 2007; 6(1): 63-65.

24. http://nodcc.org (25.08.2017).

25. Prendergast DM, Ardekani B, Ikuta T, et al. Age and sex effects on corpus callosum morphology across the lifespan. Hum Brain Mapp. 2015; 36(7): 2691-2702, doi: 10.1002/ hbm.22800, indexed in Pubmed: 25833103.

26. Renard D, Castelnovo G, Campello C, et al. An MRI review of acquired corpus callosum lesions. J Neurol Neurosurg Psychiatry. 2014; 85(9): 1041-1048, doi: 10.1136/jnnp2013-307072, indexed in Pubmed: 24563521.

27. Roy E, Hague C, Forster B, et al. The corpus callosum: imaging the middle of the road. Can Assoc Radiol J. 2014;
65(2): 141-147, doi: 10.1016/j.carj.2013.02.004, indexed in Pubmed: 23809604.

28. Salat D, Ward A, Kaye JA, et al. Sex differences in the corpus callosum with aging. Neurobiol Aging. 1997; 18(2): 191-197, indexed in Pubmed: 9258896.

29. Suganthy J, Raghuram L, Antonisamy B, et al. Gender- and age-related differences in the morphology of the corpus callosum. Clin Anat. 2003; 16(5): 396-403, doi: 10.1002/ ca.10161, indexed in Pubmed: 12903061.

30. Sullivan EV, Rosenbloom MJ, Desmond JE, et al. Sex differences in corpus callosum size: relationship to age and intracranial size. Neurobiol Aging. 2001; 22(4): 603-611, indexed in Pubmed: 11445261.

31. Tanaka-Arakawa MM, Matsui M, Tanaka C, et al. Developmental changes in the corpus callosum from infancy to early adulthood: a structural magnetic resonance imaging study. PLoS One. 2015; 10(3): e0118760, doi: 10.1371/ journal.pone.0118760, indexed in Pubmed: 25790124.

32. Takeda S, Hirashima $\mathrm{Y}$, Ikeda $\mathrm{H}$, et al. Determination of indices of the corpus callosum associated with normal aging in Japanese individuals. Neuroradiology. 2003; 45(8): 513-518, doi: 10.1007/s00234-003-1019-8, indexed in Pubmed: 12879325.

33. Weis $S$, Weber $G$, Wenger $E$, et al. The controversy about a sexual dimorphism of the human corpus callosum. Int J Neurosci. 1989; 47(1-2): 169-173, indexed in Pubmed: 2793343.

34. Witelson S. Hand and sex differences in the isthmus and genu of the human corpus callosum. A postmortem morphological study. Brain. 1989; 112(3): 799-835, doi: 10.1093/brain/112.3.799. 